\title{
Performance of surfactant-polymer flooding under the influence of intermittent ultrasonic waves
}

\author{
Nor Asyikin Noruddin *, Wan Rosli Wan Sulaiman, Abdul Razak Ismail \\ Petroleum Engineering Department, Universiti Teknologi Malaysia, Johor Bharu, Malaysia
}

\section{A R T ICLE IN F O}

\section{Article history:}

Received 2 February 2017

Received in revised form

29 August 2017

Accepted 10 September 2017

\section{Keywords:}

Intermittent ultrasonic wave

Ultrasonic wave

Surfactant-polymer

Enhanced oil recovery

Chemical flooding

\begin{abstract}
A B S T R A C T
The main focus of this research is to initiate the use of intermittent vibration in order to save the cost. Mechanical vibrations generated by ultrasonic radiation influence interfacial forces and increase the relative permeability of the phases. In this project, ultrasonic vibration was designed to assist surfactant-polymer (SP) flooding process for more significant effects, as SP flooding is one of EOR methods that is able to maximize both sweep and displacement efficiency. Besides, this work has been designed to understand the mechanics of intermittent ultrasonic vibration in influencing additional recovery of SP flooding, reduction of residual oil saturation, and displacement pattern at pore scale during the flooding process under exposure of ultrasonic waves. To achieve this, experiments consisting of visualization (at pore scale) and displacement experiments were conducted. Ultrasonic power was changed to see the influence on the process. Residual oil saturation at end of the flooding was recorded and snapshots of oil displacement were taken. The outcomes justified that intermittent vibrations can produce and enhance more additional oil recovery of SP flooding compared to the continuous vibration, and the reduction of residual oil saturation, and oil displacement rate highly dependent on the ultrasonic power.
\end{abstract}

(c) 2017 The Authors. Published by IASE. This is an open access article under the CC BY-NC-ND license (http://creativecommons.org/licenses/by-nc-nd/4.0/)

\section{Introduction}

Many mature reservoirs under water flood have low economic production rates despite having as much as $50 \%$ to $75 \%$ of the original oil still in place. In order to recovery some of the oil left in the reservoir, Enhanced Oil Recovery (EOR) has to be implemented to resolve the physical and geological effects. These mature reservoirs also are viable candidates for chemical EOR that uses both, surfactant to reduce oil/water interfacial tension (IFT) and polymer to improve sweep efficiency.

SP flooding can improve the oil recovery by introducing certain polymer and surfactant. Polymer can increase the viscosity of fluid so as to enhance the sweep efficiency, while surfactant can improve the displacement efficiency by reducing the IFT between oil and water, alternatively wettability and emulsifying crude oil.

The use of elastic wave excitation has been suggested as an alternative EOR method after the

\footnotetext{
* Corresponding Author.

Email Address: syikin.noruddin@gmail.com (N. A. Noruddin) https://doi.org/10.21833/ijaas.2017.011.013

2313-626X/@ 2017 The Authors. Published by IASE.

This is an open access article under the CC BY-NC-ND license

(http://creativecommons.org/licenses/by-nc-nd/4.0/)
}

earthquake incident in 1950's which generate elastic waves that increase water and oil production. Earthquake is one of the impulsive vibrations. Quite a number of field application and laboratory observation had been carried out to investigate the effect of continuous radiation on the rate of recovery during the past 50 years and it brings positive outcomes.

Duhon and Campbell (1965) initiated an experiment by performing water flood tests through cores under ultrasonic vibration with three different set of frequencies $(1,3.1$, and $5.5 \mathrm{MHz})$. Results from these experiments showed that the ultrasonic energy improved the recovery of oil and displacement efficiency in cores. Besides, ultrasonic excitation also resulted in the decreasing permeability of water to the ratio of oil. The permeability reduced greatly in the presence of ultrasound effect.

While, Cherskiy et al. (1977) applied pulsed ultrasonic waves to core samples saturated with fresh water and described a sharp increase in core samples permeability was observed and after stopping the ultrasound, the permeability returned to its first value. Thus, they concluded that with changing the intensity, the permeability increased by using pulsed treatment up to 10-15 times in compare with continuous sonication. 
Gadiev (1977) also did study on the effect of ultrasonic to oil saturated unconsolidated sand packs. He observed a considerable increase in oil production rate and cumulative oil production. "Sono-capillary effect" has been proposed as the phenomenon contributed to this increase in oil production. During cavitation, it is believes that the bubbles collapsed and the liquid level within a capillary is raised up. Dyblenko et al. (1989) observed the enhancement of kerosene displacement by water in a reservoir core sample by applying sound at a frequency of $200 \mathrm{~Hz}$. The mobility of residual oil increased and the permeability to water decreased during the excitation.

Hamidi (2012) pointed out that the effect of ultrasonic waves on oil recovery is more significant in oil wet especially with lower viscosities. In micromodel, they concluded that the rate of oil recovery is proportional to the ultrasound power and frequency. Recently, Mohammadian et al. (2011) studied the effects of sonication radiation on oil recovery by ultrasonic waves stimulated water-flooding. They proved that cavitation; viscosity reduction and emulsification are the mechanism involved when ultrasonic waves were applied to water flooding.

Limited work had been done in micro scale to investigate ultrasonic in terms of displacement mechanism. Li et al. (2005) performed the study on pore scale on the mobilization of oil ganglia in a 2D glass micro-model under low frequency vibration. Increased in recovery were observed with higher amplitude and lower frequency. Beresnev et al. (2005) showed vibration can solve the entrapped residual oil problem because of existing capillary forces. They concluded that residual saturation of ganglia is proportional to the amplitude and inversely proportional to the frequency.

Most of the field application and laboratory experiments involved continuous radiation and it proved the successful of enhanced oil recovery. On the other hand, the cost to generate consistence continuous radiation is rather expensive. In terms of machinery, a large amount of money need to spend in order to carried out the maintenance.

\section{Experimental procedure}

\subsection{Fluid properties}

There were three types of fluids been used throughout this study? They were oil (kerosene and brine) with viscosity of $0.4 \mathrm{cp}$ and $21 \mathrm{cp}$, respectively, 2 wt $\%$ de-aerated $\mathrm{NaCl}$ brine $(0.74 \mathrm{cp})$, and an optimum SP solution with formulation of $0.15 \mathrm{wt} \%$ AOS + 400 ppm HPAM + 20,000 ppm NaCl (6.96cp).

\subsection{Crest ultrasonic}

Constant frequency been used through the experiments which is $25 \mathrm{kHz}$. While, there were three ultrasonic intensities (150W, 300W and
$500 \mathrm{~W}$ ) were used in order to study the effect of ultrasonic power to the SP flooding efficiency. Ultrasonic vibration was applied to the porous media once SP flooding process started. Both continuous and intermittent type of wave was applied in the experiments as to compare the effectiveness of intermittent energy. Intermittent radiation been applied by manually 20 minutes switch on and 20 minutes off the ultrasonic generator, alternately (20 minutes cycle). For the continuous radiation, the ultrasonic generator was continuing to on.

\subsection{Displacement test}

Porous medium used for displacement test was artificial core with packed glass beads inside the Perspex holder (macro-model). Diameter, length and wall thickness of the macro-model was $3.4 \mathrm{~cm}, 42.5$ $\mathrm{cm}$ and $0.6 \mathrm{~cm}$ respectively. Four different ranges of glass bead sizes were used which are $90 \mu \mathrm{m}-150 \mu \mathrm{m}$, $150 \mu \mathrm{m}-250 \mu \mathrm{m}, 250 \mu \mathrm{m}-425 \mu \mathrm{m}$, and $425 \mu \mathrm{m}-600 \mu \mathrm{m}$, following ratio of $2: 1: 1: 1$. The glass beads were scattered to represent heterogeneity. Formation inside the artificial core is $30 \% \Phi$ and 1 Darcy at its best using this method due to belied that this artificial core is unconsolidated.

Displacement test were carried out to compare the recovery of SP flooding with and without the assistance of ultrasonic waves. Besides, these tests were carried out to study the influence of ultrasonic energy in enhancing the reduction of residual oil saturation, $\mathrm{S}_{\mathrm{OR}}$ after SP flooding. The effect of ultrasonic intensity (150W, 300W and 500W), and ultrasonic wave pattern (intermittent and continuous) on the performance of SP flooding through the reduction of Sor were investigated. The frequency of ultrasonic wave and injection rate was set constant at $25 \mathrm{kHz}$ and $0.5 \mathrm{ml} / \mathrm{min}$, respectively, for all displacement tests by using macro-model.

\subsection{Visualization experiments}

Porous medium used for visualization experiment was two-dimensional (2D) glass-etched (micro-model). It is designed to study on the interface of oil and water under ultrasound wave. The specification of 2D glass micro-model is shown in Table 1.

Snapshots were taken at a period of times during the displacement process took place. The injection rate used for displacement of micro-model was 1.0 $\mathrm{ml} / \mathrm{h}$. The experimental runs in this study are presented in Table 2. Figs. 1, 2, and 3 illustrate the setup of displacement test of SP flooding without and with aid of ultrasonic vibration, and visualization experiment, respectively.

Table 1: Specification of 2D glass micro-model

\begin{tabular}{cc}
\hline Parameter & Specification \\
\hline Pore volume & $37.688 \mathrm{~mm}^{3}$ \\
Dimension & $60 \mathrm{~mm} \mathrm{x} \mathrm{60mm}$ \\
Throat diameter & $0.15 \mathrm{~mm}$ \\
Porosity & $34 \%$ \\
Permeability & 1.94 Darcy \\
\hline
\end{tabular}


Table 2: Summary of experimental runs

\begin{tabular}{cccccc}
\hline No. & Test & Oil Type & Wave Type & F (kHz) & I (W) \\
\hline 1 & Displacement & Kerosene & None & None & None \\
2 & Displacement & Kerosene & CUS & 25 & 150 \\
3 & Displacement & Kerosene & CUS & 25 & 300 \\
4 & Displacement & Kerosene & CUS & 25 & 500 \\
5 & Displacement & Kerosene & IUS & 25 & 150 \\
6 & Displacement & Kerosene & IUS & 25 & 300 \\
7 & Displacement & Kerosene & IUS & 25 & 500 \\
8 & Visualization & Kerosene & CUS & 25 & 150 \\
9 & Visualization & Kerosene & CUS & 25 & 300 \\
10 & Visualization & Kerosene & CUS & 25 & 500 \\
11 & Visualization & Kerosene & IUS & 25 & 150 \\
12 & Visualization & Kerosene & IUS & 25 & 300 \\
13 & Visualization & Kerosene & IUS & 25 & 500 \\
14 & Displacement & Paraffin & CUS & 25 & 500 \\
15 & Displacement & Paraffin & IUS & 25 & 500 \\
\hline
\end{tabular}

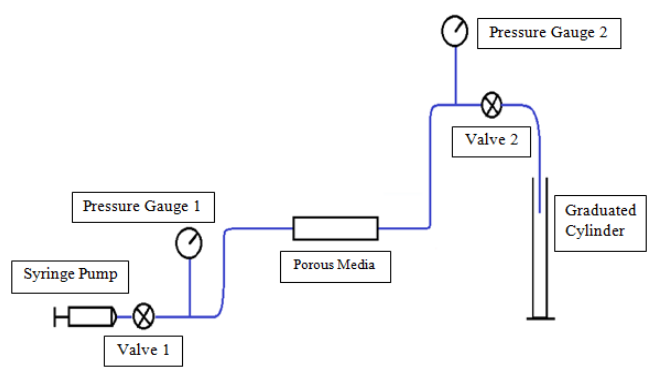

Fig. 1: Setup of SP flooding without aids of ultrasonic vibration

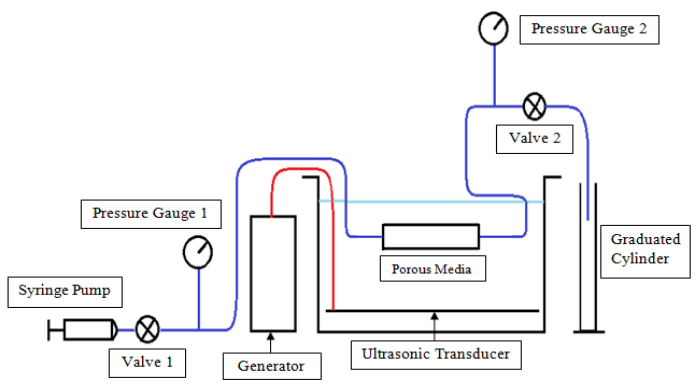

Fig. 2: Setup of SP flooding with aids of ultrasonic vibration (displacement test using macro-model)

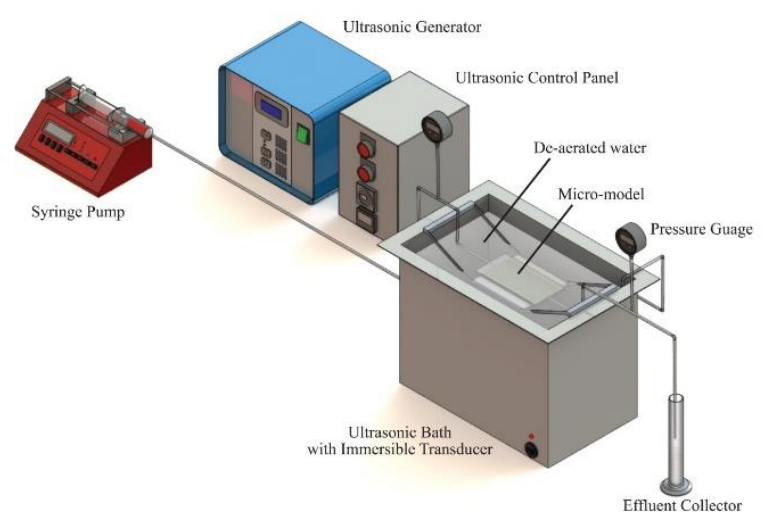

Fig. 3: Setup of visualization experiment using glass micromodel

\section{Results and discussion}

\subsection{SP flooding process assisted by ultrasonic vibration}

Displacement test was first conducted without applying any vibration energy. Purpose of conducting this experiment is to prove that ultrasound radiation can reduce the residual oil left in the porous media. Fig. 4 shows the residual oil saturation $\left(\mathrm{S}_{\mathrm{OR}}\right)$ of SP flooding with and without assisting of ultrasonic wave. Kerosene oil was used as the non-wetting phase for this process. SP slug of $0.15 \mathrm{wt} \%$ AOS + $400 \mathrm{ppm}$ HPAM + 20,000 ppm NaCl, was used to create the oil bank during the displacement process, and another 1.5PV of 20,000 ppm of $\mathrm{NaCl}$ brine solution continue to be injected as extended water flood to displace the oil at its best. Based on Fig. 4, SoR after SP flooding without aid of ultrasonic wave was $48.7 \%$. This value had been used as reference or standard guideline to compare the SP flooding process under the exposure of wave, later.

To compare the performance of SP flooding under the influence of ultrasonic waves, continuous type of vibration (CUS) been introduced with properties of $25 \mathrm{kHz}$ and $150 \mathrm{~W}$ of frequency and intensity, respectively. At the end of SP flooding process, Sor resulted to decrease for about $6 \%$ to value of $41.7 \%$.

Results from these displacement tests showed that significant improvement on oil recovery and reduction of $S_{0 R}$ had been achieved when ultrasonic waves were exposed to porous media during the SP flooding process.

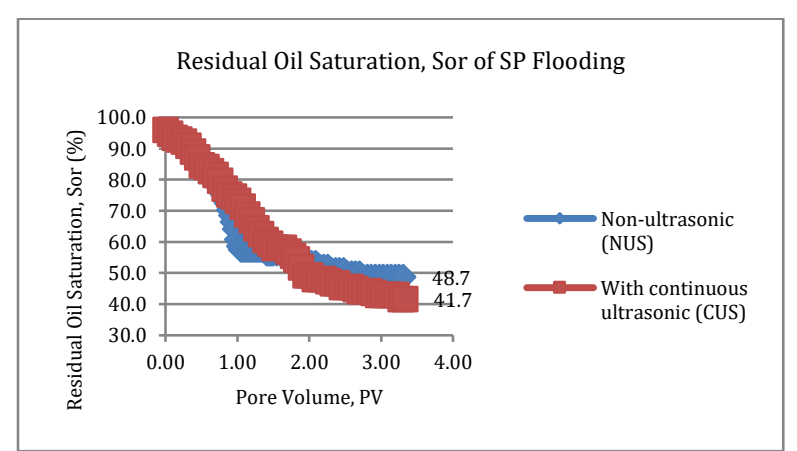

Fig. 4: Residual oil saturation, SoR of SP flooding with and without assistance of ultrasonic wave

\subsection{SP flooding assisted by intermittent (IUS) and continuous (CUS) wave}

Fig. 5 shows the results comparison Sor after exposing to IUS and CUS. Frequency of wave used was $25 \mathrm{kHz}$ with $150 \mathrm{~W}$ of intensity. SP flooding assisted by IUS left $39.6 \%$ of oil inside the porous media compared to CUS which is $41.7 \%$.

For the visualization experiments, snapshots were taken during the process of SP flooding. Fig. 6 shows the comparison of micro-model displacement experiment between IUS and CUS for Kerosene oil (light oil).

Red color represents oil, while white color represents water. The snapshots were taken once the SP flooding process were started as the ultrasonic vibration been on. On the application of IUS, there was lesser oil left inside the micro-model at the end of the process compared to CUS. Results from these experiments showed that IUS improved 
the recovery of oil and increased rate of oil displacement. In addition, ultrasonic radiation enhanced the movement of oil in porous media and hence, reduced the Sor.

Results showed intermittent vibration can solve the entrapped residual oil problem during SP flooding process more effectively than continuous vibration.

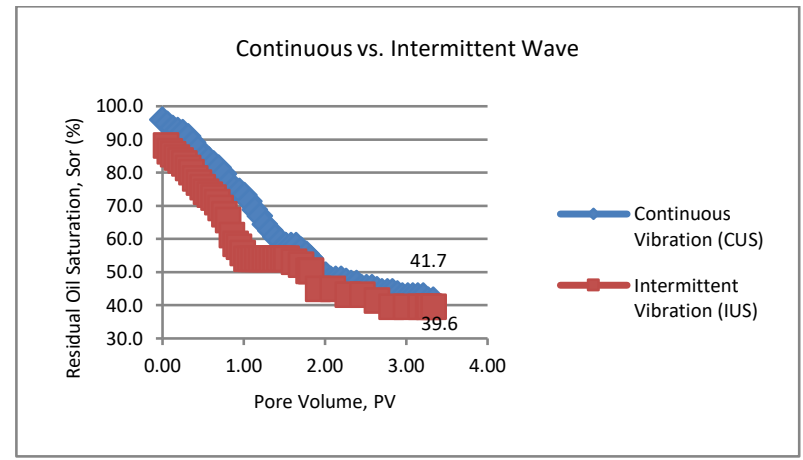

Fig. 5: Results comparison of residual oil left in porous medium after exposing to IUS and CUS

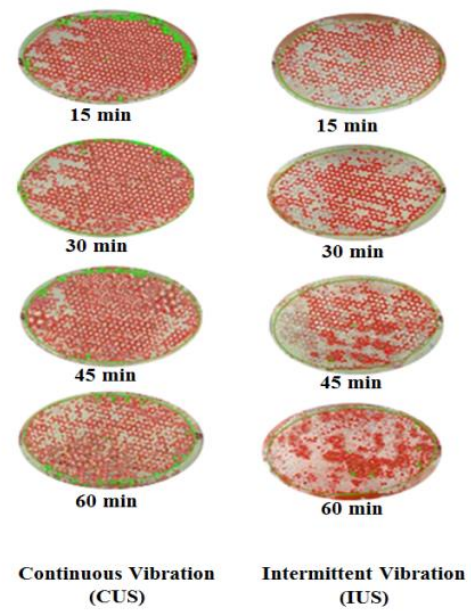

Fig. 6: Comparison of micro-model displacement experiment between IUS and CUS for light oil application

\subsection{Effect of Ultrasonic Intensity on SP Flooding Recovery}

Figs. 7 and 8 show the effect of ultrasonic power intensity (150W, 300W and 500W) on the Sor after SP flooding for light oil application. For continuous vibration (CUS), there were very small decrements of Sor recorded as the ultrasonic intensity applied was increased. Sor recorded was $39.0 \%$ when maximum ultrasonic intensity (500W) applied for CUS. Total decrement of Sor recorded was about $9.7 \%$ as compared to without vibration (NUS) and 500W intensity of CUS.

While, for intermittent vibration (IUS), there was $4.6 \%$ decrement of SoR when ultrasonic intensity was increased from $150 \mathrm{~W}$ to $500 \mathrm{~W}$. Total decrement of Sor recorded was about $13.7 \%$ as compared to without vibration (NUS) and 500W intensity of IUS.

As for conclusion, IUS contributed to large decrement of Sor when increasing the ultrasonic intensity compared to CUS. Results obtained can be summarized into the decrement of $\mathrm{S}_{\mathrm{OR}}$ is proportional to the ultrasound power and IUS able to reduce the residual oil left in porous media in a significant value of percentage.

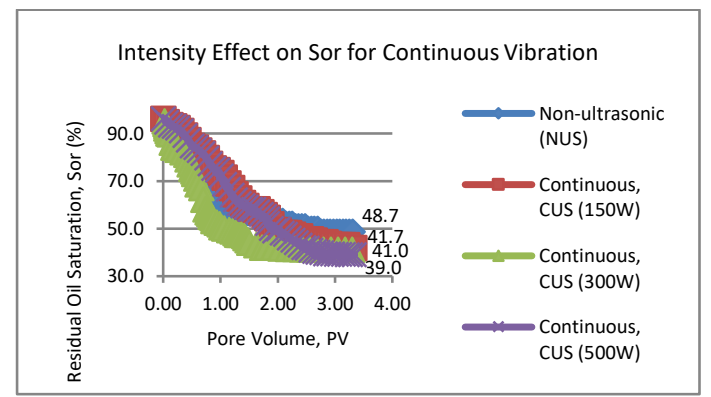

Fig. 7: Effect of ultrasonic intensity of continuous vibration (CUS) on Sor after SP flooding

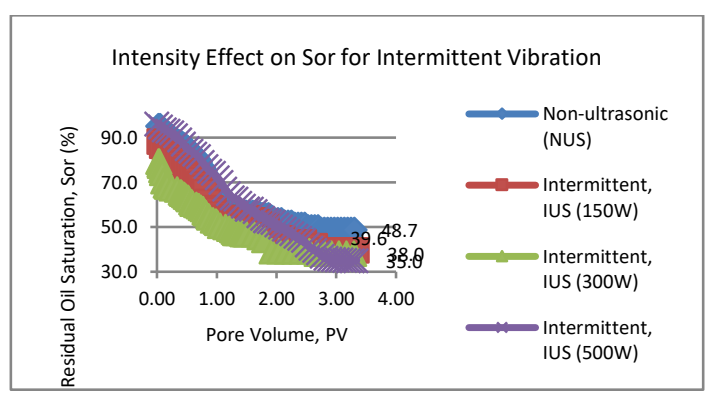

Fig. 8: Effect of ultrasonic intensity of interittent vibration (IUS) on Sor after SP flooding

For the visualization experiments, Figs. 9 and 10 show the effect of ultrasonic power on micro-model displacement experiment between CUS and IUS for light oil application, respectively. Both CUS and IUS resulted in the reduction of $S_{O R}$ as the intensity used getting higher. But, only slight changes (reduction of red fluid) can be observed for oil displacement using CUS. Besides, for the application of IUS, there was significant reduction of $\mathrm{S}_{\mathrm{O}}$ has been observed for all the three intensities used. It shows that oil less trap in the micro-model compared to the CUS. Red fluid (oil) moved faster in higher intensity as IUS radiation enhanced the movement of oil in porous media and hence Sor. Mechanism involved here is the coalescence of 2 or more oil droplets into the larger droplets having higher mobility then become part of the flow stream due to the Bjerknes forces (attractive forces acting between the vibrating droplets). Interfacial tension had been reduced and will affect the capillary number. Capillary number will be increased and residual will be decreased. This mean more oil can be extracted from the pore.

\subsection{Effect of ultrasonic power on heavy oil recovery of SP flooding}

Fig. 11 shows the results of $\mathrm{S}_{\mathrm{OR}}$ of Paraffin oil (represent heavy oil) under the influence of continuous (CUS) and intermittent (IUS) waves. SoR recorded for CUS was $53.9 \%$.

While, Sor for IUS was $47.0 \%$. So, there were about $6.9 \%$ of $\mathrm{S}_{\mathrm{OR}}$ can be decreased by using IUS. Heavy oil contributed higher percent of decrement as compared to light oil (4\% decrement). Results 
from these displacement tests can be concluded that IUS effect on reduction of $S_{0 R}$ is more significant for heavier oil application.

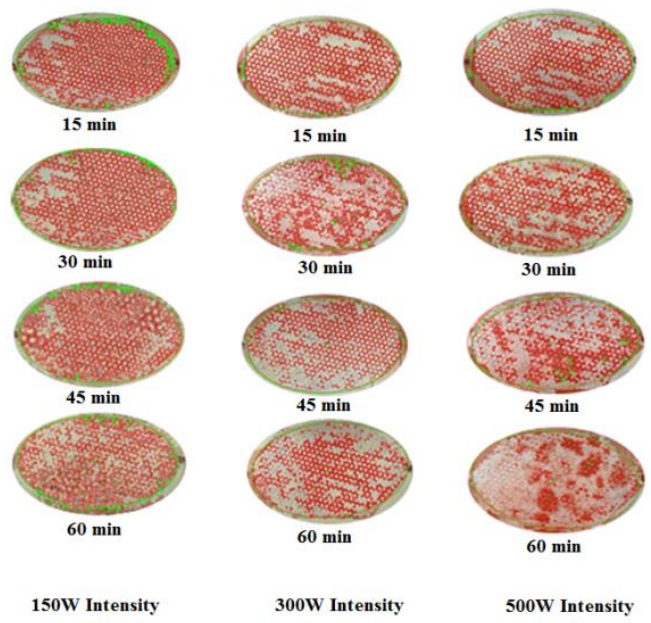

Fig. 9: Intensity effect on the pattern of oil displacement (continuous vibration, CUS)
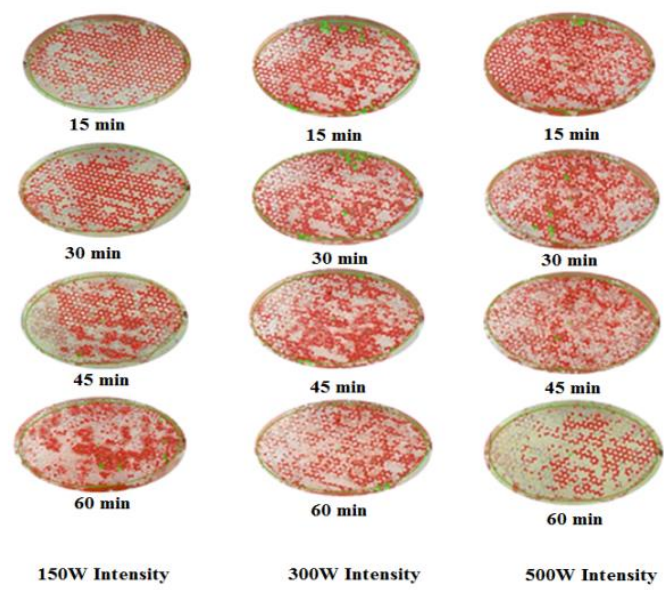

Fig. 10: Intensity effect on the pattern of oil displacement (intermittent vibration, IUS)

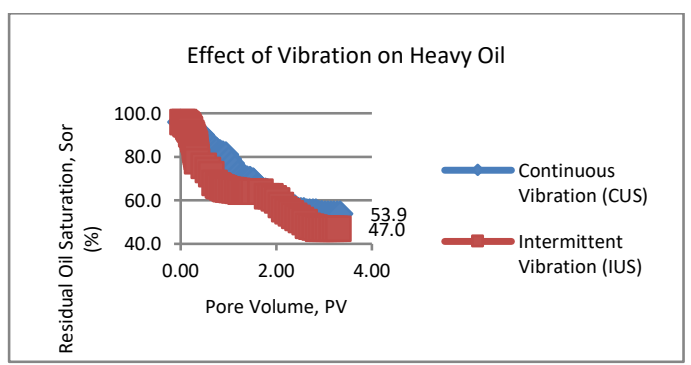

Fig. 11: Effect of vibration on heavy oil application

Fig. 12 shows the displacement pattern of heavy oil using continuous and intermittent vibration. As can be observed, IUS works compatible with heavy oil. Heavy oil contains different high molecules and it starts to break up after ultrasonic vibration is applied. Due to its relative movements of the molecules and difference in their acceleration leads to reduction in the oil viscosity and producing more oil. When oil viscosity is reduced, mobility ratio will be decreased. These experiments concluded that intermittent vibration work well in high oil viscosity and compatible for heavy oil application.

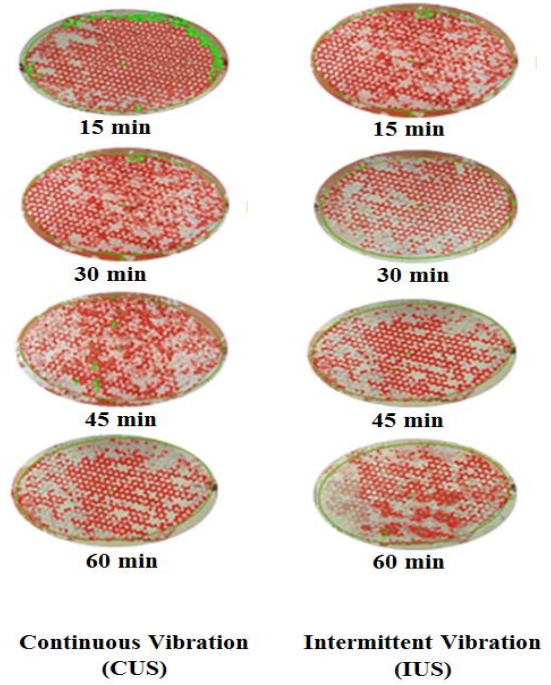

Fig. 12: Oil displacement pattern for heavy oil application under the influence of CUS and IUS

\section{Conclusion}

Series of displacement experiments were conducted in this study and the following conclusions were made: (1) ultrasonic vibration proved to enhance a significant value of additional oil recovery on SP flooding; (2) Intermittent ultrasonic wave, IUS, managed to produce more oil recovery compared to continuous vibration; (3) Intermittent ultrasonic wave, IUS, together with high intensity of ultrasonic gives the best recovery and reduces the $S_{O R}$ at the very best value; (4) Intermittent ultrasonic wave, IUS, also compatible with high viscosity of oil and SP solution; and (5) vibration can reduce the IFT and oil viscosity to allow residual oil to move out from the reservoir.

\section{Acknowledgement}

This work was funded by the Grant No. 00K60 (with Grant Ref. No PY/2014/03342) and Grant No. 00 M93 (with Grant Ref. No. of PY/2015/04612). The authors appreciate the Faculty of Petroleum and Renewable Energy Engineering at Universiti Teknologi Malaysia in Johor Bahru, Malaysia, for providing Laboratory facilities in completing this work.

\section{References}

Beresnev I, Vigil D, and Li W (2005). The mechanism of recovery of residual oil by elastic waves and vibrations. SEG Technical Program Expanded Abstracts, 24: 1386-1389. https://doi.org/10.1190/1.2147946

Cherskiy NV, Tsarev VP, Konovalov VM, and Kuznetsov OL (1997). The effect of ultrasound on permeability of rocks to water. Transactions (Doklady) of the USSR Academy of Sciences, Earth Science Section, 232: 201-204.

Duhon RD and Campbell JM (1965). The effect of ultrasonic energy on flow through porous media. In the SPE $2^{\text {nd }}$ Annual Eastern Regional Meeting of SPE/AIME, Society of Petroleum Engineers, Charleston, USA: 1-15. https://doi.org/10.2118/ 1316-MS 
Dyblenko VP, Tufanov IA, Suleymanov GA, and Lysenkov AP (1989). Percolation phenomena and processes in saturated porous media under the vibro-wave action, in ways of intensification of oil production. (Putu intensifikatsii dobychi nefti). Proc. (Trudy) Basharki Res. Design Inst. Oil (Bashnipineft): 45-51. [In Russian].

Gadiev SM (1977). Use of vibrations in oil production. Nedra Press, Moscow, Russia.

Hamidi H (2012). A role of ultrasonic frequency and power on oil mobilization in underground petroleum reservoirs. Journal of Petroleum Exploration and Production Technology, 2(1): 2936.
Li W, Vigil RD, Beresnev IA, Iassonov P, and Ewing R (2005). Vibration-induced mobilization of trapped oil ganglia in porous media: Experimental validation of a capillary-physics mechanism. Journal of Colloid and Interface Science, 289(1): 193-199.

Mohammadian E, Shirazi MA, and Idris AK (2011). Enhancing oil recovery through application of ultrasonic assisted waterflooding. In the SPE Asia Pacific Oil and Gas Conference and Exhibition, Society of Petroleum Engineers, Jakarta, Indonesia: 1-10. https://doi.org/10.2118/145014-MS 